\title{
Pressure-Induced Phase Transformations, Electronic Properties and Intermediate Phases of Chromium Dioxide
}

\author{
H. ÖZTÜRK ${ }^{a}$ AND C. KÜRKÇÜ ${ }^{b, *}$ \\ ${ }^{a}$ Department of Physics, Ahi Evran University, Kırşehir, 40100, Turkey \\ ${ }^{b}$ Department of Electronics and Automation, Ahi Evran University, Kırşehir, 40100, Turkey \\ (Received October 29, 2017; revised version March 11, 2019; in final form July 18, 2019)
}

\begin{abstract}
We study pressure-induced phase transformations of chromium dioxide $\left(\mathrm{CrO}_{2}\right)$ using constant pressure $a b$ initio simulations. A first-order phase transformation from the rutile-type structure to the $\mathrm{CaCl}_{2}$-type structure is successfully observed at $220 \mathrm{GPa}$. Simulations reveal that this transformation proceeds via two intermediate phases within $P \overline{4} 2_{1} m$ and $P 2_{1} 2_{1} 2$ symmetries. Another phase transformation from this structure to the orthorhombic structure with space group $C m c 2_{1}$ occurs at $440 \mathrm{GPa}$. This phase transformation also proceeds via two intermediate phases within $P 2_{1} / c$ and $P 2_{1}$ symmetries. We also study these phase transformations of $\mathrm{CrO}_{2}$ by undertaking the enthalpy calculations. We find that the phase transformation from the rutile-type structure to the $\mathrm{CaCl}_{2}-$ type structure occurs around $15 \mathrm{GPa}$ which is in good agreement with the experiments. Also, the phase transformation from $\mathrm{CaCl}_{2}$-type to the orthorhombic structure with space group $C m c 2_{1}$ is found at $105 \mathrm{GPa}$. Additionally, we predict band gaps from electronic structure calculations for obtained phases of $\mathrm{CrO}_{2}$
\end{abstract}

DOI: 10.12693/APhysPolA.136.26

PACS/topics: chromium dioxide, high pressure, phase transformation, intermediate phase

\section{Introduction}

Chromium dioxide $\left(\mathrm{CrO}_{2}\right)$ has attracted specific interest as a compound possessing near $100 \%$ spin polarization at the Fermi level. Its half-metallic performance leads to relatively low electrical resistivity for an oxide. It is also ferromagnetic at room temperature relative to the other half-metal candidates. These properties and wide availability of $\mathrm{CrO}_{2}$ make this material technologically and scientifically noteworthy and a perfect substance for developing spintronic devices [1-11].

This material is regarded as a perfect candidate for enhancing spintronic devices, tunneling magnetoresistance devices, magnetic heads, magnetic random access memories, and magnetic field sensors. Due to the uniaxial crystal structure, $\mathrm{CrO}_{2}$ is estimated to have a great magnetic anisotropy which makes it a preferable material for magneto-optical recording [3, 12-21].

$\mathrm{CrO}_{2}$ crystallizes in a tetragonal rutile-type structure with space group $\mathrm{P}_{2} / \mathrm{mnm}$ at ambient conditions like $\mathrm{TiO}_{2}, \mathrm{MnO}_{2}, \mathrm{RuO}_{2}, \mathrm{SnO}_{2}, \mathrm{GeO}_{2}$, etc. Among these rutile-type dioxides, $\mathrm{CrO}_{2}$ has exceptional meaning because $\mathrm{CrO}_{2}$ is the only half-metallic ferromagnet. A phase transformation from the tetragonal rutiletype to the $\mathrm{CaCl}_{2}$-type structure is observed by Maddox et al. [12] at $12 \pm 3 \mathrm{GPa}$.

We have also found this phase transformation at about $15 \mathrm{GPa}$ by ab initio calculations in good agreement with their study. We also suggest that this orthorhombic structure with space group Pnnm of $\mathrm{CrO}_{2}$ proceeds through two intermediate phases with space

*corresponding author; e-mail: ckurkcu@ahievran.edu.tr groups $P \overline{4} 2_{1} m$ and $P 2_{1} 2_{1} 2$, expressed as $\mathrm{CrO}_{2}$-(a) and $\mathrm{CrO}_{2}$-(b), respectively. To our knowledge, these intermediary phases of this material have not been found in any earlier studies. Additionally, we have observed a new phase transformation of this material from the $\mathrm{CaCl}_{2}$ type structure to an orthorhombic structure with space group $C m c 2_{1}$ at about $105 \mathrm{GPa}$. This orthorhombic structure also proceeds through two intermediate phases with space group $P 2_{1} / c$ and $P 2_{1}$, expressed as $\mathrm{CrO}_{2}$-(c) and $\mathrm{CrO}_{2}-(\mathrm{d})$, respectively. This orthorhombic structure has not been recognized for $\mathrm{CrO}_{2}$ before, to the best of our knowledge.

\section{Computational details}

The calculations in the present work were carried out in the frame of density-functional theory (DFT) with the ab initio program SIESTA [22]. Local density approximation (LDA) using the Ceperley-Alder functional [23] was used for the exchange-correlation energy. Double- $\xi$ plus polarized basis sets were employed and a uniform mesh with a plane wave cut-off of 200 Ry was used. A chosen simulation cell consists of 96 atoms with periodic boundary conditions. We employed $\Gamma$-point sampling for the Brillouin zone integration. To get optimized lattice vectors and atomic positions, the stress tolerance and maximum atomic force were taken less than $0.5 \mathrm{GPa}$ and smaller than $0.01 \mathrm{eV} \AA^{-1}$, respectively. Firstly, the system was equilibrated at zero pressure, and after that the pressure was gradually increased by an increment of $20 \mathrm{GPa}$ applying conjugate gradient method to the system. In order to find symmetries of the phases observed in the simulations, we used the RGS algorithm and the KPLOT program $[24,25]$ that permitted detailed data about a given structure such as space group, cell parameters, and atomic positions. 
The relative stability of the different phases of $\mathrm{CrO}_{2}$ under finite pressure and temperature can be easily identified by a simple comparison of their Gibbs free energies, $G=E_{\text {tot }}+P V-T S$. Since calculations in this study are achieved at zero temperature, the last term is ignored. As a result, the static enthalpy can be expressed as $H=E_{\text {tot }}+P V$, where pressure is acquired by direct differentiation of the energy-volume curves i.e., $P=-\mathrm{d} E_{\mathrm{tot}} / \mathrm{d} V[26]$. The rutile-type, $\mathrm{CaCl}_{2}$-type and orthorhombic structures were equilibrated at several volumes and their energy-volume relations were fitted to the third-order Birch-Murnaghan equation of state [27, 28]. For the energy-volume calculations, the Brillouin zone integration was achieved with an automatically created 6-6-6 $k$-point mesh for three phases of $\mathrm{CrO}_{2}$ succeeding the convention of Monkhorst and Pack [29]. Enthalpy calculations supply generally accurate transition pressures comparable to experiments. These pressures can be easily found by matching the enthalpy of the phases for the reason that the phase transitions emerge at equal enthalpy of the two phases transformed one to another one.

\section{Results and discussions}

Initially, we relax a 96 atoms supercell at zero pressure to obtain the lattice parameters of the $P 4_{2} / \mathrm{mnm}$ phase and afterwards compare them with available literature data. The equilibrium unit cell lattice constants of $\mathrm{CrO}_{2}$ are obtained as $a=4.3754 \AA$, and $c / a=0.5927$. These values are comparable with the previous experimental results of $a=4.4190$ $4.4320 \AA$ and $c / a=0.6568-0.6597[12,16,19,21]$ and the theoretical results of $a=4.3840-4.4560 \AA$ and $c / a=0.6349-0.6597[18,30,31]$.

The pressure-volume relation of $\mathrm{CrO}_{2}$ is investigated and presented in Fig. 1. The volume gives sharp variations when the applied pressure is increased from $200 \mathrm{GPa}$ to $220 \mathrm{GPa}$, and from $420 \mathrm{GPa}$ to $440 \mathrm{GPa}$ indicating pressure-induced phase transitions in this material. The structural analysis reveals that the tetragonal rutile-type structure transforms to the orthorhombic $\mathrm{CaCl}_{2}$-type structure at $220 \mathrm{GPa}$ and this $\mathrm{CaCl}_{2}$-type structure transforms to another orthorhombic structure at $440 \mathrm{GPa}$, as shown in Fig. 2. Their equilibrium lattice parameters and the atomic fractional coordinates are summarized in Table I. In order to examine whether there are another phase transformations or not, we have increased the pressure up to $500 \mathrm{GPa}$. We have not observed any other phase for $\mathrm{CrO}_{2}$.

The obtained phase transition pressure values using gradually increasing pressure technique show that the $a b$ initio technique successfully produces the experimentally observed high-pressure phase of $\mathrm{CrO}_{2}$ with an overvalued transition pressure. Since the restricted conditions such as finite size of the simulation cell, the lack of any defect in simulated structure, etc. are considered,

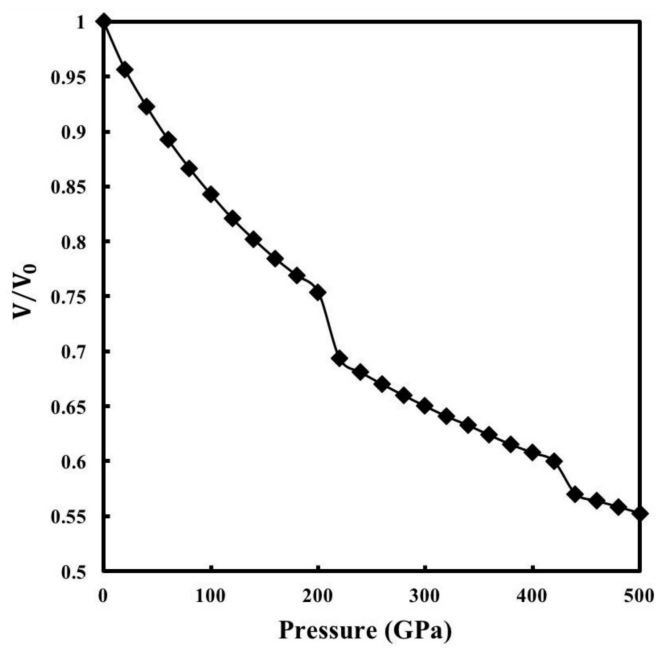

Fig. 1. The volume change of the simulation cell as function of pressure.

(a)

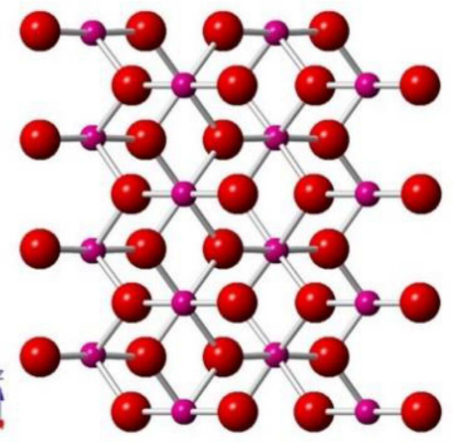

(b)

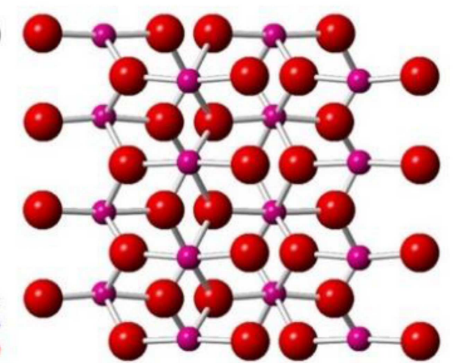

(c)

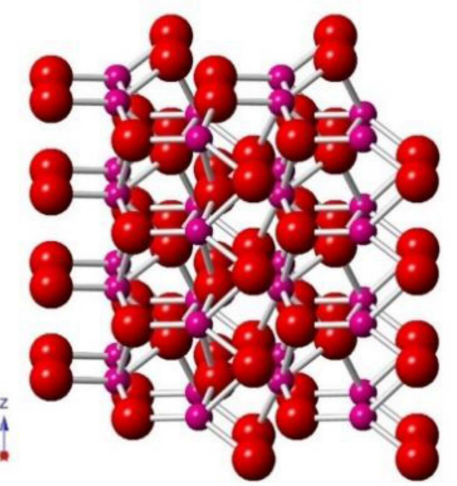

Fig. 2. Crystal structures of $\mathrm{CrO}_{2}$ : (a) $\mathrm{P} 4_{2} / \mathrm{mnm}$ at zero pressure, (b) Pnnm at $220 \mathrm{GPa}$, and (c) $C m c 2_{1}$ at $440 \mathrm{GPa}$. 
Equilibrium lattice parameters and atomic fractional coordinates of three main phases of $\mathrm{CrO}_{2}$

TABLE I

\begin{tabular}{c|c|c|c|c|c|c}
\hline \hline Phase & $a[\AA]$ & $b[\AA]$ & $c[\AA]$ & $x$ & $y$ & $z$ \\
\hline$P 4_{2} /$ mnm & 4.3754 & 4.3754 & 2.5932 & Cr: 0.5000 & 0.5000 & 0.5000 \\
& & & & O: 0.7890 & 0.7890 & 0.5000 \\
& & & & O: 0.2110 & 0.2110 & 0.5000 \\
\hline Pnnm & 3.7145 & 4.2312 & 2.1888 & Cr: 0.5000 & 0.5000 & 0.5000 \\
& & & & O: 0.3136 & 0.1437 & 0.5000 \\
& & & & O: 0.6864 & 0.8563 & 0.5000 \\
\hline Cmc2 $2_{1}$ & 2.0887 & 7.4108 & 3.6589 & Cr: 0.0000 & 0.3813 & 0.0001 \\
& & & & O: 0.0000 & 0.9548 & 0.6684 \\
& & & & O: 0.5000 & 0.4548 & 0.6684
\end{tabular}

TABLE II

Transition pressures, equilibrium lattice parameters, equilibrium volume rates, bulk modulus and their pressure derivatives of three main phases of $\mathrm{CrO}_{2}$.

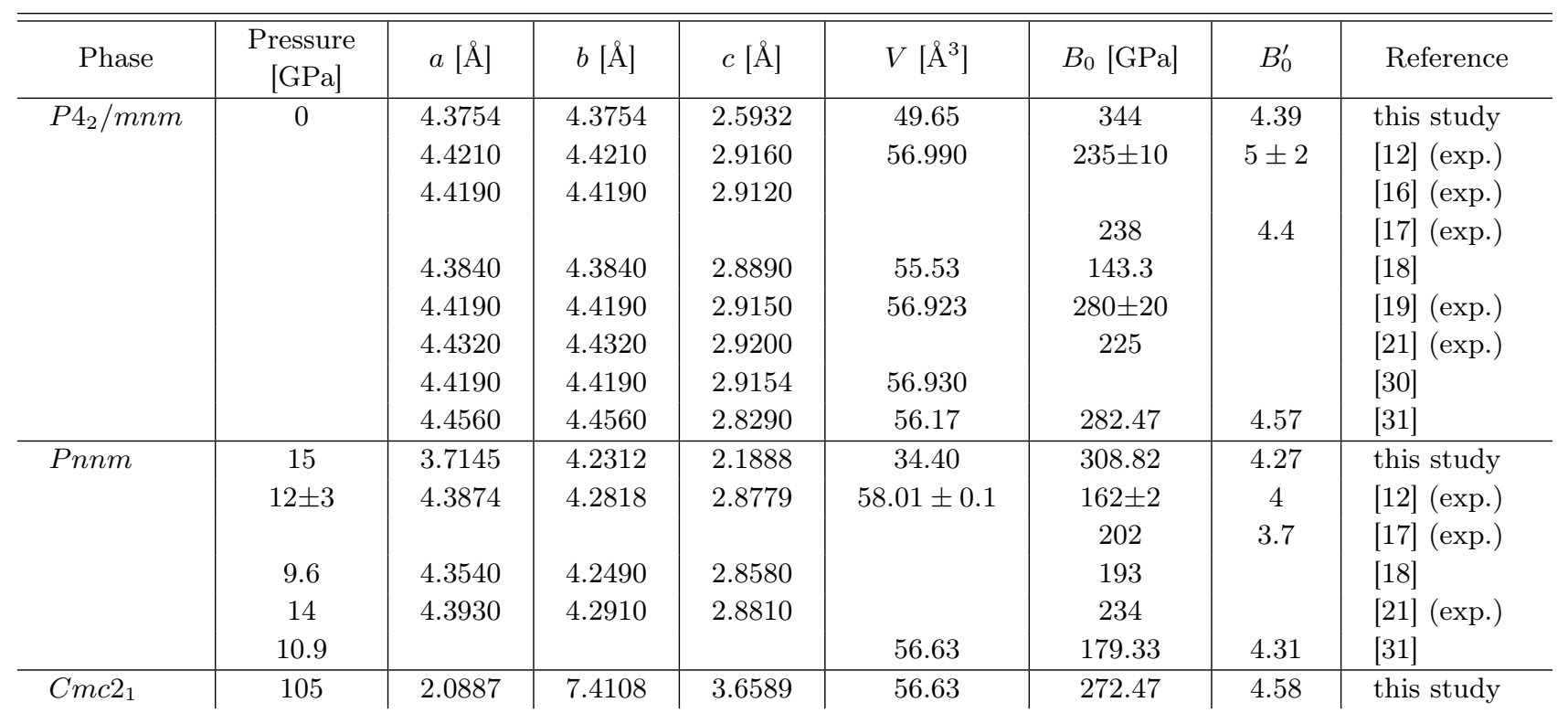

such an overestimated transition pressure is expected [32]. Conversely, the thermodynamic theorem does not take into account the probable presence of an activation barrier separating the two structural phases. Thus, as a next step, we take the energy-volume computations into account to study the stability of highpressure phases of $\mathrm{CrO}_{2}$. For these computations we used the unit cells for the $P 4_{2} / m n m$, Pnnm, and $C m c 2_{1}$ high-pressure phases. Both of the unit cells of the first two phases have 6 atoms and the unit cell of the last phase has 12 atoms.

The third-order Birch-Murnaghan equation of state, given by

$$
\begin{aligned}
P & =1.5 B_{0}\left[\left(\frac{V}{V_{0}}\right)^{-\frac{7}{3}}-\left(\frac{V}{V_{0}}\right)^{-\frac{5}{3}}\right] \\
& \times\left\{1+0.75\left(B_{0}^{\prime}-4\right)\left[\left(\frac{V}{V_{0}}\right)^{-\frac{2}{3}}-1\right]\right\},
\end{aligned}
$$

was used to fit the energy-volume data for three main structures of $\mathrm{CrO}_{2}$. Here $P$ is the pressure, $V$ is the volume at pressure, $V_{0}, B_{0}$, and $B_{0}^{\prime}$ are the volume, bulk modulus, and its first pressure derivate at $0 \mathrm{GPa}$, respectively [27, 28]. The computed total energies as a function of volumes are presented in Fig. 3. The equilibrium lattice parameters, equilibrium volume rates, bulk modulus, and their pressure derivatives, together with the other theoretical and experimental data for three main structures of $\mathrm{CrO}_{2}$ are reported in Table II. The bond lengths between $\mathrm{Cr}$ and $\mathrm{O}$ atoms with minimum and maximum values and coordination numbers of the $P 4_{2} / m n m, P \overline{4} 2_{1} m, P 2_{1} 2_{1} 2$, Pnnm, $P 2_{1} / c, P 2_{1}$, and $C m c 2_{1}$ phases are given in Table III and the calculated density and porosity data of these phases are given in Table IV.

The phase transition pressure between different phases of $\mathrm{CrO}_{2}$ is easily identified by a simple comparison of their static lattice enthalpy since the intersection of two enthalpy curves indicates a pressure-induced phase 
transition between these two phases. The computed enthalpy curves of the $P 4_{2} / m n m$, Pnnm, and $C m c 2_{1}$ high-pressure phases are plotted as a function of pressure in Fig. 4. As seen from this figure the enthalpy curve of the $P 4_{2} / \mathrm{mnm}$ phase crosses with that of the Pnnm phase at about $15 \mathrm{GPa}$ indicating phase transition between these phases and the enthalpy curve of the Pnnm phase crosses with that of the $C m c 2_{1}$ phase at about $105 \mathrm{GPa}$ between these phase couple indicating another phase transition.

In order to evaluate transformation mechanism at the atomistic level, we investigate the variations of the simulation cell vectors and angles as a function of minimization step. Figure 5 displays the modification of the simulation cell lengths and angles at 220 and $440 \mathrm{GPa}$, respectively. These simulation cell vectors are called as $\boldsymbol{A}$, $\boldsymbol{B}$, and $\boldsymbol{C}$ which are initially along the [100], [010], and [001] directions, respectively.

\section{TABLE III}

The bond lengths with minimum and maximum values and coordination numbers of the $P 4_{2} / \mathrm{mnm}, P \overline{4} 2_{1} m$, $P 2_{1} 2_{1} 2, P n n m, P 2_{1} / c, P 2_{1}$ and $C m c 2_{1}$ phases. $B_{\text {min }}$ and $B_{\max }$ indicate minimum and maximum bond lengths, respectively.

\begin{tabular}{c|c|c|c}
\hline \hline Phase & $B_{\min }[\AA]$ & $B_{\max }[\AA]$ & \multicolumn{1}{c}{ Coordination } \\
\hline$P 4_{2} / m n m$ & 1.773 & 1.863 & $\mathrm{Cr} \Longrightarrow[2-6] \mathrm{O}$ \\
$P \overline{4} 2_{1} m$ & 1.571 & 1.722 & $\begin{array}{l}\mathrm{O} \Longrightarrow[1-3] \mathrm{Cr} \\
\mathrm{Cr} \Longrightarrow[2-6] \mathrm{O}\end{array}$ \\
$P 2_{1} 2_{1} 2$ & 1.580 & 1.763 & $\begin{array}{l}\mathrm{O} \Longrightarrow[1-3] \mathrm{Cr} \\
\mathrm{Cr} \Longrightarrow[2-6] \mathrm{O}\end{array}$ \\
$P n n m$ & 1.641 & 1.743 & $\begin{array}{l}\mathrm{O} \Longrightarrow[1-3] \mathrm{Cr} \\
\mathrm{Cr} \Longrightarrow[2-6] \mathrm{O}\end{array}$ \\
$P 2_{1} / c$ & 1.565 & 1.702 & $\begin{array}{l}\mathrm{O} \Longrightarrow[1-3] \mathrm{Cr} \\
\mathrm{Cr} \Longrightarrow[2-6] \mathrm{O}\end{array}$ \\
$P 2_{1}$ & 1.565 & 2.164 & $\begin{array}{l}\mathrm{Or} \Longrightarrow[1-3] \mathrm{Cr} \\
\mathrm{O} \Longrightarrow[2-7] \mathrm{O}\end{array}$ \\
$C m c 2_{1}$ & 1.615 & 1.725 & $\begin{array}{l}\mathrm{Cr} \Longrightarrow[2-7] \mathrm{Cr} \\
\mathrm{O} \Longrightarrow[1-4] \mathrm{Cr}\end{array}$
\end{tabular}

TABLE IV

The calculated density and porosity data of the $P 4_{2} / m n m, P \overline{4} 2_{1} m, P 2_{1} 2_{1} 2$, Pnnm, $P 2_{1} / c, P 2_{1}$ and $C m c 2_{1}$ phases.

\begin{tabular}{l|c|c|c|c|c}
\hline \hline Phase & $\begin{array}{c}\text { Calculated } \\
\text { density } \\
{\left[\mathrm{kg} / \mathrm{m}^{3}\right]}\end{array}$ & $\begin{array}{c}\text { Unit cell } \\
\text { volume } \\
{\left[\AA^{3}\right]}\end{array}$ & $\begin{array}{c}\text { Atom } \\
\text { density } \\
\left.\text { [atoms } / \AA^{3}\right]\end{array}$ & $\begin{array}{c}\text { Filled } \\
\text { space } \\
{\left[\AA^{3}\right]}\end{array}$ & $\begin{array}{c}\text { Void } \\
\text { space } \\
{\left[\AA^{3}\right]}\end{array}$ \\
\hline$P 4_{2} / m n m$ & 1758.7308 & 2537.9583 & 0.0378 & 529.204 & 2008.754 \\
$P \overline{4} 2_{1} m$ & 2400.0535 & 1859.7859 & 0.0516 & 509.143 & 1350.643 \\
$P 2_{1} 2_{1} 2$ & 2334.0608 & 1912.3689 & 0.0502 & 508.221 & 1404.148 \\
$P n n m$ & 2628.0095 & 1698.4662 & 0.0565 & 504.234 & 1194.233 \\
$P 2_{1} / c$ & 2884.3579 & 1547.5144 & 0.0620 & 479.873 & 1067.642 \\
$P 2_{1}$ & 2431.8752 & 1835.4500 & 0.0523 & 475.683 & 1359.767 \\
$C m c 2_{1}$ & 2080.3394 & 2145.6045 & 0.0447 & 478.085 & 1667.520
\end{tabular}

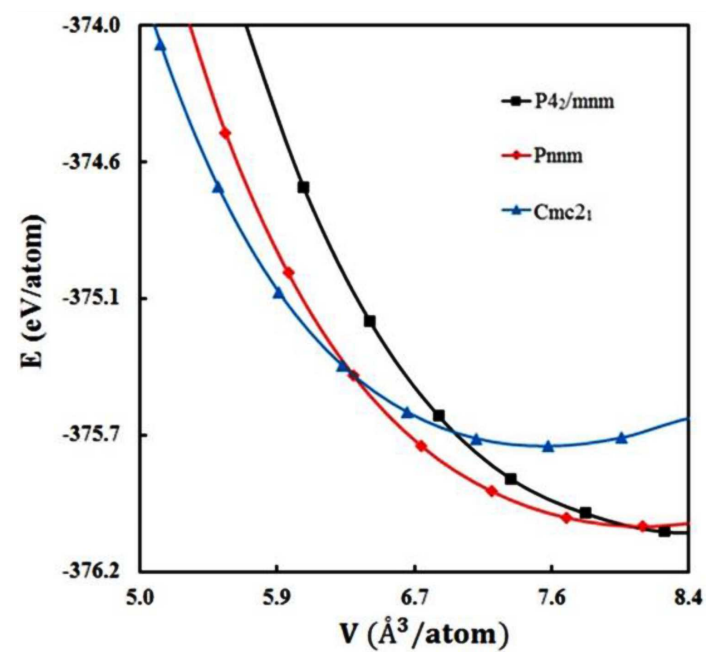

Fig. 3. The energy-volume curves of main structural phases of $\mathrm{CrO}_{2}$.

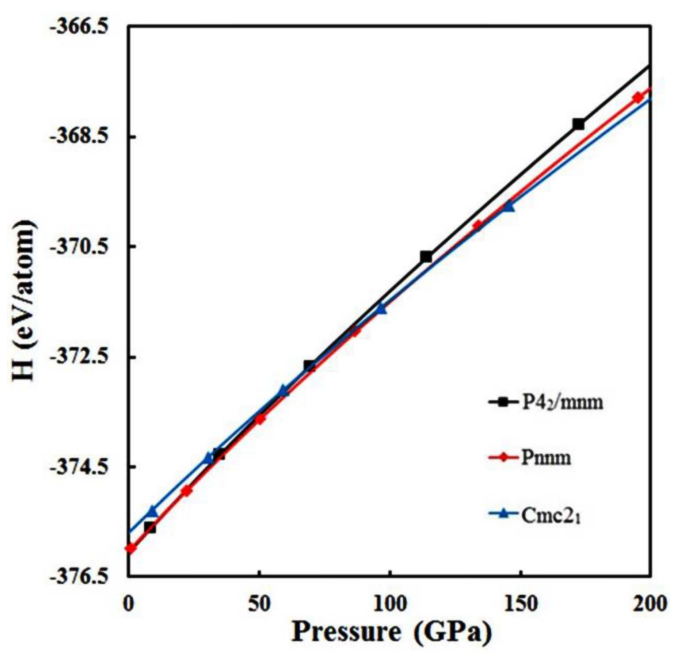

Fig. 4. The enthalpy curves of main structural phases of $\mathrm{CrO}_{2}$ as function of pressure.

In order to investigate whether there is any intermediate state during the phase transformation or not, we analyzed the obtained structures in this study at each minimization step by using KPLOT program. For the Pnnm phase of $\mathrm{CrO}_{2}$, we determined a tetragonal structure with $\mathrm{P}_{2} / \mathrm{mnm}$ symmetry at $34^{\text {th }}$ minimization step and an orthorhombic structure with $P 2_{1} 2_{1} 2$ symmetry at $43^{\text {rd }}$ minimization step. The orthorhombic $\mathrm{CaCl}_{2}$-type structure having Pnnm symmetry forms at $71^{\text {st }}$ minimization step. These intermediate states are depicted in Fig. 6. For the $C m c 2_{1}$ phase of $\mathrm{CrO}_{2}$, we observed a monoclinic structure with $P 2_{1} / c$ symmetry at $33^{\text {rd }}$ minimization step and another monoclinic structure with $P 2_{1}$ symmetry at $99^{\text {th }}$ minimization step. The orthorhombic structure having $C m c 2_{1}$ symmetry forms at $130^{\text {th }}$ minimization step. These intermediary states are depicted in Fig. 7. 
(a)
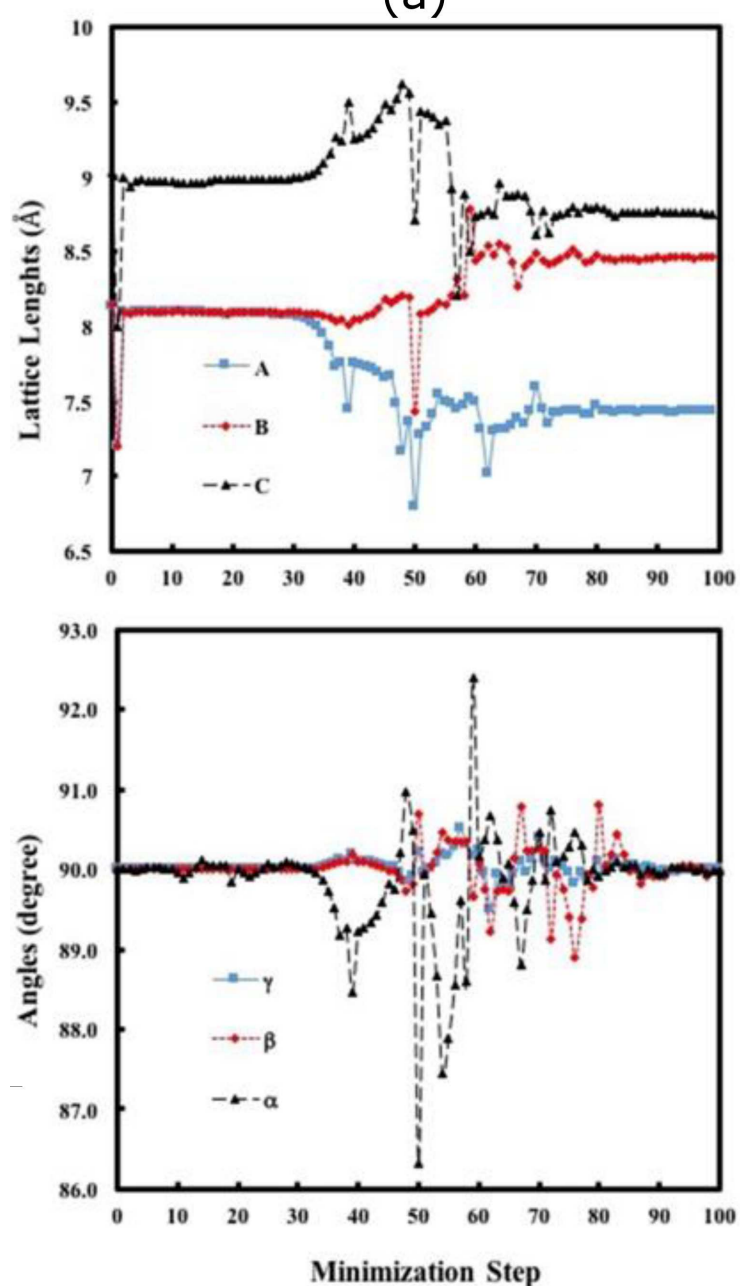

(b)
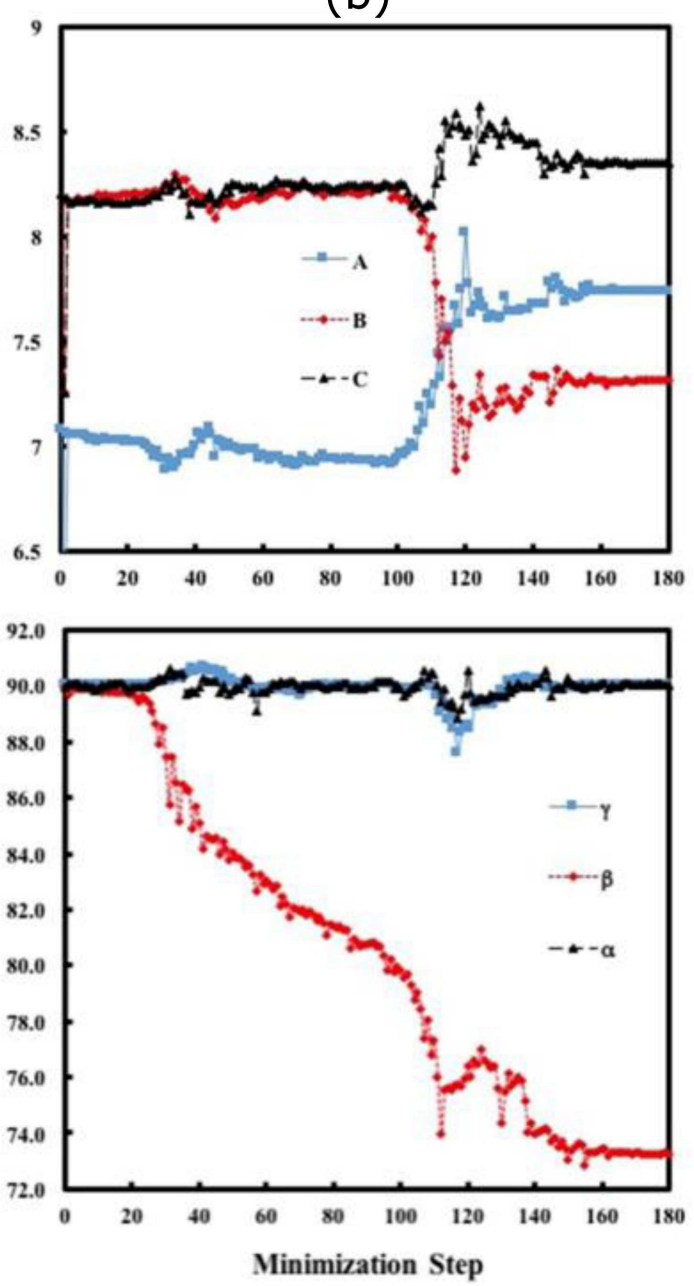

Fig. 5. The behavior of the simulation cell lengths and angles as function of minimization steps: (a) at 220 GPa and

(b) at $440 \mathrm{GPa}$.

(a)

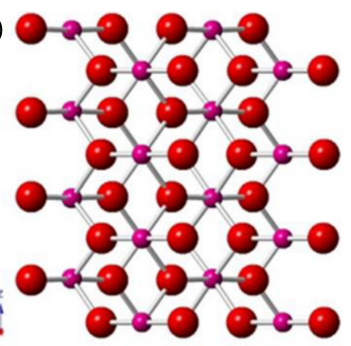

(b) $0-20-2$

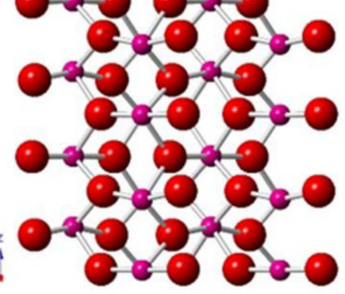

(c)

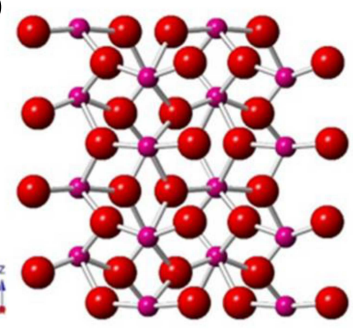

(d)

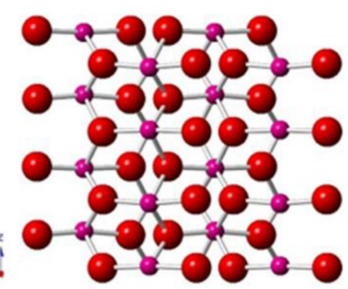

Fig. 6. Evolution of the Pnnm phase at $220 \mathrm{GPa}$ :

(a) $P 4_{2} / m n m$, (b) $P \overline{4} 2_{1} m$, (c) $P 2_{1} 2_{1} 2$, and (d) Pnnm. (a)

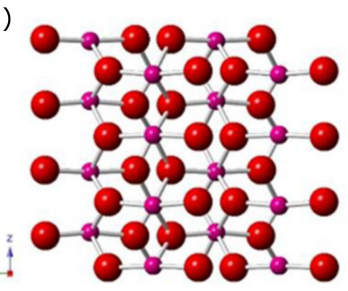

(c)

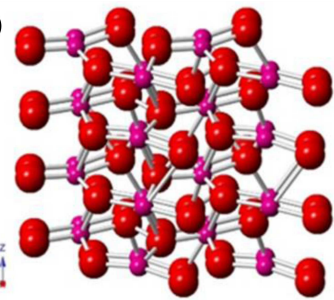

(b)

(d)

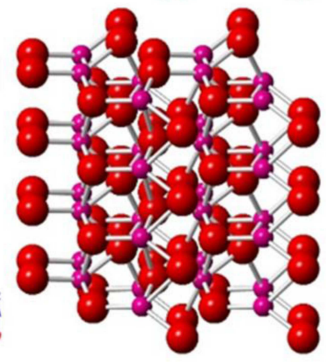

Fig. 7. Evolution of the $C m c 2_{1}$ phase at $440 \mathrm{GPa}$ : (a) Pnnm, (b) $P 2_{1} c$, (c) $P 2_{1}$, and (d) $C m c 2_{1}$. 
We calculated the band structures with minority and majority spin states along the high-symmetry directions for $P 4_{2} / \mathrm{mnm}$, Pnnm, and $C m c 2_{1}$ phases of $\mathrm{CrO}_{2}$. Figures 8-10 illustrate band structures of main phases of $\mathrm{CrO}_{2}$ near the Fermi energy $\left(E_{\mathrm{F}}\right)$ level at $0 \mathrm{GPa}, 220 \mathrm{GPa}$ and $440 \mathrm{GPa}$ pressure, respectively. The Fermi levels were set to be $0 \mathrm{eV}$. The symmetry points were $\Gamma, X, M, \Gamma, Z, R, A$, and $M$ for $P 4_{2} / m n m$ phase, $\Gamma, Z, T, Y, \Gamma, X, S, R$, and $U$ for Pnnm phase, and $\Gamma, Z, T, Y, \Gamma, S, R$, and $Z$ for $C m c 2_{1}$ phase. In Fig. 8 , the electronic band curves for the spin up state show that $P 42 / m n m$ phase of $\mathrm{CrO}_{2}$ has metallic character. In the event of spin down state, there is a direct band gap because the valence band maximum and conduction band minimum are situated at the same point $(\Gamma$-point). Since the band curves obtained for spin up and spin down states do not overlap, the $\mathrm{P}_{2} / \mathrm{mnm}$ phase of $\mathrm{CrO}_{2}$ also has a magnetic property. In Fig. 9, according to the obtained band curves, it is obvious that the Pnnm phase of $\mathrm{CrO}_{2}$ has metallic properties as they cut the Fermi level. In Fig. 10, Cmc2 1 phase of $\mathrm{CrO}_{2}$ is similar to the semiconductor character having a direct band gap at $S$-point. However, since the band curves for the spin up and spin down states overlap, the Pnnm and $C m c 2_{1}$ phases of $\mathrm{CrO}_{2}$ do not have magnetic properties.

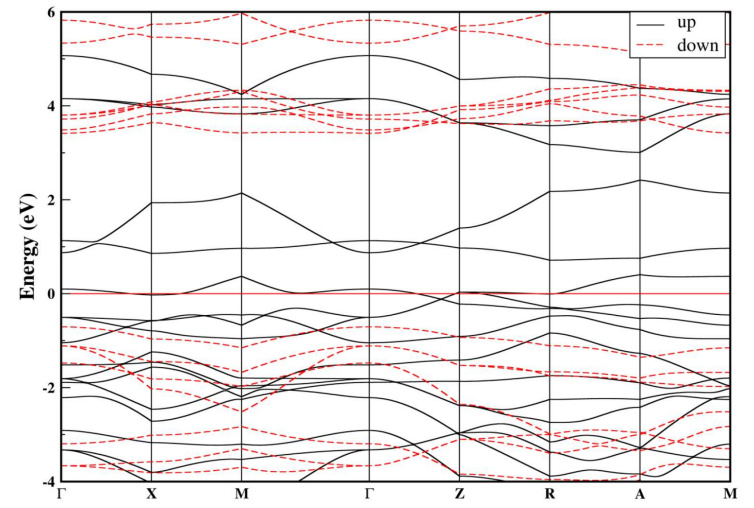

Fig. 8. The calculated spin polarized band structures of $\mathrm{CrO}_{2}$ at $0 \mathrm{GPa}$.

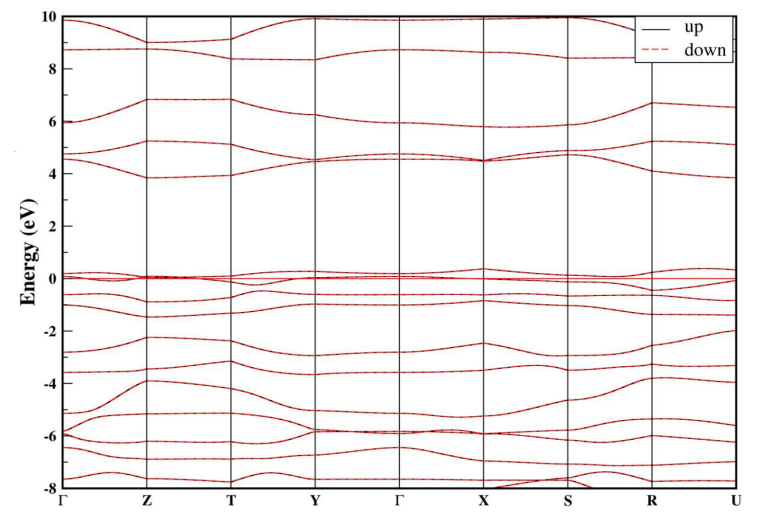

Fig. 9. The calculated spin polarized band structures of $\mathrm{CrO}_{2}$ at $220 \mathrm{GPa}$.

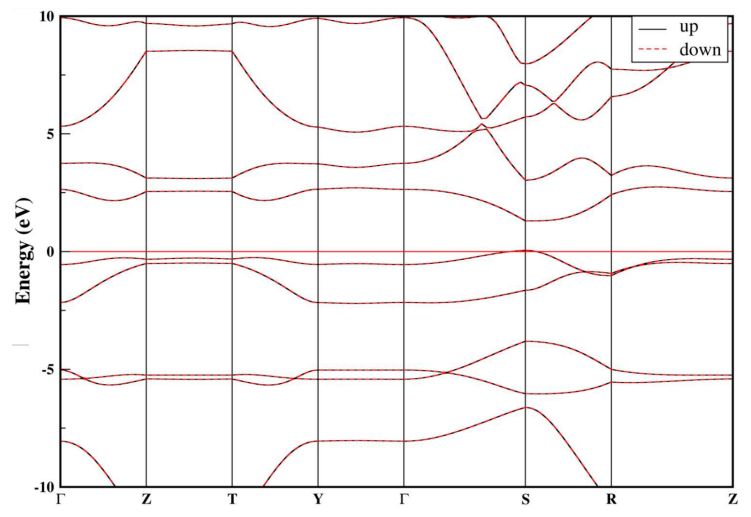

Fig. 10. The calculated spin polarized band structures of $\mathrm{CrO}_{2}$ at $440 \mathrm{GPa}$.

\section{Conclusions}

We have performed an $a b$ initio constant pressure technique in order to study the pressure-induced phase transformation in the rutile-type structured chromium dioxide. The phase transition path is obtained as $\mathrm{P}_{2} / \mathrm{mnm} \rightarrow \mathrm{Pnnm} \rightarrow \mathrm{Cmc2}_{1}$. Two intermediary states are observed for both phase transitions. These intermediary states and the orthorhombic structure with space group $C m c 2_{1}$ of $\mathrm{CrO}_{2}$ have not been observed in any other previous studies to our knowledge. Additionally, we calculated the electronic band structure of $\mathrm{CrO}_{2}$. From these calculations, it can be seen that all phases have metallic character. In addition, the $P 4_{2} / \mathrm{mnm}$ phase of $\mathrm{CrO}_{2}$ shows a magnetic property while the other high pressure phases do not have magnetic properties. Our findings allow different viewpoints on the transformation mechanism of the rutile-type structure to the $\mathrm{CaCl}_{2}$-type structure phase change and that of $\mathrm{CaCl}_{2}$ type structure to the orthorhombic structure with space group $C m c 2_{1}$.

\section{Acknowledgments}

This study was supported by the Ahi Evran University Scientific Research Projects Coordination Unit. Project Number: FEF.A3.16.026.

\section{References}

[1] Y. Ji, G.J. Strijkers, F.Y. Yang, C.L. Chien, J.M. Byers, A. Anguelouch, G. Xiao, A. Gupta, Phys. Rev. Lett. 86, 5585 (2001).

[2] J.S. Parker, S.M. Watts, P.G. Ivanov, P. Xiong, Phys. Rev. Lett. 88, 196601 (2002).

[3] K.P. Kämper, W. Schmitt, G. Güntherodt, R.J. Gambino, R. Ruf, Phys. Rev. Lett. 59, 2788 (1987)

[4] Y.S. Dedkov, M. Fonin, C. König, U. Rüdiger, G. Güntherodt, S. Senz, D. Hesse, Appl. Phys. Lett. 80, 4181 (2002). 
[5] J.M.D. Coey, A.E. Berkowitz, L.I. Balcells, F.F. Putris, A. Barry, Phys. Rev. Lett. $\mathbf{8 0}$, 3815 (1998).

[6] J.M.D. Coey, S. Sanvito, J. Phys. D Appl. Phys. 37, 988 (2004).

[7] D.J. Huang, L.H. Tjeng, J. Chen, C.F. Chang, W.P. Wu, S.C. Chung, A. Tanaka, G.Y. Guo, H-J. Lin, S.G. Shyu, Phys. Rev. B 67, 214419 (2003).

[8] C.B. Stagarescu, X. Su, D.E. Eastman, K.N. Altmann, F.J. Himpsel, A. Gupta, Phys. Rev. B 61, R9233 (2000).

[9] E.Z. Kurmaev, A. Moewes, S.M. Butorin, M.I. Katsnelson, L.D. Finkelstein, J. Nordgren, P.M. Tedrow, Phys. Rev. B 67, 155105 (2003).

[10] M.N. Iliev, A.P. Litvinchuk, H.G. Lee, C.W. Chu, A. Barry, J.M.D. Coey, Phys. Rev. B 60, 33 (1999).

[11] K. Schwarz, J. Phys. F Met. Phys. 16, L211 (1986)

[12] B.R. Maddox, C.S. Yoo, D. Kasinathan, W.E. Pickett, R.T. Scalettar, Phys. Rev. B 73, 144111 (2006).

[13] R.M. Hazen, L.W. Finger, J. Phys. Chem. Solids 42, 143 (1981).

[14] J. Haines, J.M. Léger, S. Hoyau, J. Phys. Chem. Solids 56, 965 (1995).

[15] V. Kanchana, G. Vaitheeswaran, M. Alouani, J. Phys. Condens. Matter 18, 5155 (2006).

[16] A.Y. Kuznetsov, J.S. De Almeida, L. Dubrovinsky, R. Ahuja, S.K. Kwon, I. Kantor, A. Kantor, N. Guignot, J. Appl. Phys. 99, 53909 (2006).

[17] V. Srivastava, M. Rajagopalan, S.P. Sanyal, Europ. Phys. J. B 61, 131 (2008)
[18] S.F. Matar, G. Demazeau, Chem. Phys. Lett. 407, 516 (2005).

[19] V.A. Sidorov, A.V. Rakhmanina, O.A. Morya, Solid State Commun. 139, 360 (2006).

[20] Y. Li, J. Hao, Solid State Commun. 152, 1216 (2012).

[21] P.I. Sorantin, K. Schwarz, Inorg. Chem. 31, 567 (1992).

[22] P. Ordejón, E. Artacho, J.M. Soler, Phys. Rev. B 53, R10441 (1996).

[23] D.M. Ceperley, B.J. Alder, Phys. Rev. Lett. 45, 566 (1980).

[24] A. Hannemann, R. Hundt, J.C. Schön, M. Jansen, J. Appl. Crystallogr. 31, 922 (1998)

[25] R. Hundt, J.C. Schön, A. Hannemann, M. Jansen, J. Appl. Crystallogr. 32, 413 (1999).

[26] M. Durandurdu, J. Phys. Condens. Matter 20, 325232 (2008).

[27] F. Birch, Phys. Rev. 71, 809 (1947).

[28] F.D. Murnaghan, Proc. Natl. Acad. Sci. 30, 244 (1944).

[29] H.J. Monkhorst, J.D. Pack, Phys. Rev. B 13, 5188 (1976).

[30] H.Y. Wu, Y.H. Chen, C.R. Deng, X.F. Su, Phase Transit. 85, 708 (2012).

[31] S. Alptekin, J. Mol. Model. 21, 1 (2015).

[32] R. Martoňák, A. Laio, M. Parrinello, Phys. Rev. Lett. 90, 075503 (2003). 